\title{
SOME APPLICATIONS OF THE STONE-WEIERSTRASS
} THEOREM

\author{
P. J. DE PAEPE
}

AbSTRACT. Let $A$ be a function algebra on a compact Hausdorff space $X$ and let $f \in A$. The Stone-Weierstrass theorem is used to obtain results on the function algebra on $X$ generated by the elements of $A$ and the function $\bar{f}$.

Preliminaries. If $A$ is a collection of continuous functions on a compact Hausdorff space $X$ separating the points of $X,[A ; X]$ denotes the function algebra on $X$ generated by the elements of $A$, i.e., the smallest algebra of continuous functions on $X$ containing the elements of $A$ and the constant functions which is uniformly closed in $C(X)$, i.e., closed in the algebra $C(X)$ of all continuous functions on $X$ provided with the supremum norm $\|\cdot\|_{X}:\|f\|_{X}=\sup \{|f(x)|: x \in X\}$. If $f$ (respectively $A$ ) is a function (a collection of functions) on $X$, and $Y$ is a subset of $X$, then $f \mid Y(A \mid Y)$ denotes the restriction of $f$ to $Y$ (the collection of restrictions of elements of $A$ to $Y$ ).

If $A$ is a function algebra on $X$, a closed subset $K$ of $X$ is called a peak set for $A$ if there exists a function $f \in A$ such that $f(K)=\{1\}$ and $|f(x)|<1$ for all $x \in X \backslash K$. If $K$ is a peak set for $A$ then $A \mid K$ is closed in $C(K)$ [3, p. 163].

A subset $K$ of $X$ is called a set of antisymmetry for $A[2$, p. 60] if any $f \in A$ which is real-valued on $K$ is constant on $K$. The collection of maximal sets of antisymmetry is a closed pairwise disjoint cover of $X$. The generalized Stone-Weierstrass theorem reads:

$$
\begin{aligned}
& \text { If } f \in C(X) \text { and } f|K \in A| K \text { for all maximal sets of } \\
& \text { antisymmetry } K \text { for } A \text {, then } f \in A \text {. }
\end{aligned}
$$

If $X$ is a compact subset of the complex plane, $P(X)$ is the function algebra on $X$ consisting of uniform limits on $X$ of polynomials; $R(X)$ is the function algebra on $X$ consisting of uniform limits on $X$ of rational functions with pole sets missing $X$.

The algebra $A(X)$ is the function algebra on $X$ consisting of all continuous functions on $X$ which are holomorphic on the interior of $X$.

Finally the complex homomorphism space of a function algebra $A$ is denoted by $\Delta A$. We abbreviate for $Y$ closed in $\Delta A:[A \mid Y]=[A \mid Y ; Y]$.

In the following we determine the function algebra $[A, f ; X]$ where $A$ is a

Received by the editors June 1, 1977.

AMS (MOS) subject classifications (1970). Primary 46J10, 46J15.

Key words and phrases. Function algebra, Stone-Weierstrass theorem, set of antisymmetry. 
function algebra on $X$ and $f$ is a real-valued function on $X$ or the complex conjugate of an element of $A$. As corollaries we obtain results of Mergelyan, Minsker and Preskenis.

THEOREM 1. Let $A$ be a function algebra on $X, f$ a real-valued continuous function on $X$. Let $X_{\alpha}=\{x \in X: f(x)=\alpha\}, \alpha \in f(X)$. Then $[A, f ; X]=$ $\left\{g \in C(X): g \mid X_{\alpha} \in\left[A \mid X_{\alpha}\right]\right.$ for each $\left.\alpha \in f(X)\right\}$.

Proof. The inclusion " $\subset$ " is trivial. Conversely, let $K$ be a maximal set of antisymmetry for $[A, f ; X]$, then $K \subset X_{\alpha_{0}}$ for some $\alpha_{0}$. Any $g \in C(X)$ such that $g \mid X_{\alpha} \in\left[A \mid X_{\alpha}\right]$ for each $\alpha$ has the property that $g \mid X_{\alpha_{0}} \in\left[A \mid X_{\alpha_{0}}\right] \subset$ $\left[[A, f ; X] \mid X_{\alpha_{0}}\right]=[A, f ; X] \mid X_{\alpha_{0}}$. The last equality follows from the fact that $X_{\alpha_{0}}$ is a peak set for $[A, f ; X]$. So also $g|K \in[A, f ; X]| K$, so by the StoneWeierstrass theorem $g \in[A, f ; X]$.

An immediate consequence is the following result of Mergelyan [4].

ThEOREM 2 (MERGELYAN). Let $X$ be a compact subset of $\mathbf{C} ; f$ a real-valued continuous function on $X$ such that $X_{\alpha}=\{x \in X: f(x)=\alpha\}$ is polynomially convex for each $\alpha \in f(X)$. Then $[z, f ; X]=[R(X), f ; X]$ and this function algebra consists of all elements of $C(X)$ which are holomorphic on the interior of the sets $X_{\alpha}$.

Proof. Let $A=P(X)$ and apply Theorem 1. By the classical Mergelyan theorem $\left[A \mid X_{\alpha}\right]=P\left(X_{\alpha}\right)=A\left(X_{\alpha}\right)$. Trivially $[z, f ; X] \subset[R(X), f ; X] \subset\{g$ $\in C(X): g$ is holomorphic on the interior of the sets $\left.X_{\alpha}\right\}$.

THEOREM 3. Let $A$ be a function algebra on $X, f \in A$. Then $[A, \bar{f} ; X]=\{g$ $\in C(X): g \mid X_{\alpha} \in\left[A \mid X_{\alpha}\right]$ for each $\left.\alpha \in f(X)\right\}$, where $X_{\alpha}=\{x \in X: f(x)=$ $\alpha\}, \alpha \in f(X)$.

Proof. Again the inclusion " $C$ " is trivial. Since both $\operatorname{Re} f$ and $\operatorname{Im} f$ belong to $[A, \bar{f} ; X], X_{\alpha}$ is the intersection of the peak sets for $[A, \bar{f} ; X]:\{x \in$ $X: \operatorname{Re} f(x)=\operatorname{Re} \alpha\},\{x \in X: \operatorname{Im} f(x)=\operatorname{Im} \alpha\}$, so is itself a peak set for $[A, \bar{f} ; X]$. Also if $K$ is a maximal set of antisymmetry for $[A, \bar{f} ; X], K$ is contained in some $X_{\alpha}$ (since both $\operatorname{Re} f$ and $\operatorname{Im} f$ are constant on $K$ ). The conclusion now follows as in the proof of Theorem 1.

Applying this result to the algebra $R(X), X \subset \mathrm{C}$, we obtain

THEOREM 4. Let $X$ be a compact subset of the complex plane.

(i) Let $g \in R(X)$ and let $X_{\alpha}=\{x \in X: g(x)=\alpha\}, \alpha \in g(X)$. Then $[R(X), \bar{g} ; X]=\left\{f \in C(X): f \mid X_{\alpha} \in R\left(X_{\alpha}\right)\right.$ for each $\left.\alpha \in g(X)\right\}$.

(ii) Let $g \in R(X)$ such that the level sets $X_{\alpha}$ of $g$ are polynomially convex. Then $[R(X), \bar{g} ; X]$ consists of all elements of $C(X)$ which are holomorphic on the interior of the sets $X_{\alpha}$.

In particular:

(iii) If $X$ is polynomially convex and $g \in P(X)$ then $[z, \bar{g} ; X]$ consists of all elements of $C(X)$ which are holomorphic on the interior of the level sets of $g$.

Proof. For a compact subset $Y$ of $X,[R(X) \mid Y]=R(Y)$ if and only if $Y$ is 
$R(X)$-convex. Since $X_{\alpha}$ is $R(X)$-convex $\left[R(X) \mid X_{\alpha}\right]=R\left(X_{\alpha}\right)$. Now apply Theorem 3 and (i) follows. Using the classical Mergelyan theorem (ii) and (iii) follow immediately.

THEOREM 5. Let $X$ be a compact set of the complex plane.

(i) Let $g \in R(X)$ such that the level sets $X_{\alpha}$ of $g$ are polynomially convex and such that $g$ is not constant on any of the components of the interior of $X$. Then $[R(X), \bar{g} ; X]=C(X)$.

In particular (Preskenis [6]):

(ii) If $X$ is polynomially convex and $g \in P(X)$ such that $g$ is not constant on any of the components of the interior of $X$ then $[z, \bar{g} ; X]=C(X)$.

This result follows immediately from Theorem 4 since the hypothesis on $g$ implies that the interior of the sets $X_{\alpha}$ are empty. An abstract version of Theorem 5(ii) is

THEOREM 6. Let $A$ be a function algebra on $X$. Let $f \in A$ and let $Y$ be the polynomially convex hull of $f(X)$. Let $g \in P(Y)$ such that $g$ is not constant on any of the components of the interior of $Y$. Then $[A, \bar{f} ; X]=[A, \bar{g} \circ f ; X]$.

Proof. Since $g \in C(Y), \bar{g}$ is uniformly approximable on $Y$ by polynomials in $z$ and $\bar{z}$, so the inclusion " $\supset$ " follows since $\bar{g} \circ f \in[A, \bar{f} ; X]$. Conversely, by Theorem 5(ii) it follows that $[z, \bar{g} ; Y]=C(Y)$, hence $\bar{z} \in[z, \bar{g} ; Y]$ so $\bar{f} \in[A, \bar{g} \circ f ; X]$.

Applying Theorem 5(ii) we obtain a result of Minsker [5]:

COROLlary 1 (MinsKer). Let $X$ be a compact subset of the complex plane and $m \in \mathbf{N}$. Then $\left[z, \bar{z}^{m} ; X\right]=C(X)$.

COROLlary 2. Let $f$ and $g$ be holomorphic on a neighborhood of $0 \in \mathbf{C}$ such that $\partial f / \partial z(0) \neq 0$ and $g$ is not constant near 0 . Then there exists a closed disc $D$ centered at the origin such that $[f, \bar{g} ; D]=[g, \bar{f} ; D]=C(D)$.

Proof. Without loss of generality $f(0)=0$, so if $\delta>0$ is small enough $z$ can be approximated uniformly on $D=\{|z| \leqslant \delta\}$ by polynomials in $f$. Moreover we may assume that $g$ is defined on $D$. By Theorem 5(ii) $[z, \bar{g}$; $D]=C(D)$, so $[f, \bar{g} ; D]=C(D)$. And $[g, \bar{f} ; D]=[g, \bar{z} ; D]$ consists of the complex conjugates of the elements of $[\bar{g}, z ; D]$. So $[g, \bar{f} ; D]=C(D)$.

Corollary 3. Let $X=\{1 \leqslant|z| \leqslant 2\} \subset \mathbf{C}$ and let $r_{1}, r_{2} \in R(X)$ such that $r_{1} \notin P(X)$ and $r_{2}$ is not constant on $X$. Then $\left[z, r_{1}, \bar{r}_{2} ; X\right]=C(X)$.

Proof. Since $P(X)$ is maximal in $R(X)[1],\left[z, r_{1} ; X\right]=R(X)$ and by Theorem 5(i) $\left[R(X), \bar{r}_{2} ; X\right]=C(X)$.

Using techniques similar to those in the previous section we prove two other results.

TheOREM 7. Let $n, m \in \mathrm{N}$ such that $\operatorname{gcd}(n, m)=1$ and let $D=\{|z| \leqslant 1\}$. If $f$ is a nonconstant element of $P(D)$, then $\left[z^{n}, z^{m}, \bar{f} ; D\right]=C(D)$. 
Proof. There exists $N \in \mathbf{N}$ such that $z^{k} \in A=\left[z^{n}, z^{m} ; D\right]$ for each $k \geqslant N$. Without loss of generality we may assume $f(0)=0$, so $f^{N}$ is uniformly approximable on $D$ by elements of $A$.

Therefore $\operatorname{Re} f^{N}$ and $\operatorname{Im} f^{N}$ belong to $[A, \bar{f} ; D]$. Let $K$ be a maximal set of antisymmetry for $[A, \bar{f} ; D]$. Then $K$ is contained in a level set $L$ of $f^{N}$ which is a peak set for $[A, \bar{f} ; D]$. Since $f^{N}$ is not constant on $D, L=P \cup\left\{a_{1}, a_{2}\right.$, $\left.a_{3}, \ldots\right\}$ where $P$ is a proper subset of $\{|z|=1\}$ and where $\left\{a_{1}, a_{2}, a_{3}, \ldots\right\}$ is a discrete subset of $\{|z|<1\}$. Now $\Delta A=D$. (Indeed: let $\phi \in \Delta A$ such that $\phi\left(z^{N}\right)=0$. If $k \in \mathbf{N}$ such that $z^{k} \in A$ then $\left(\phi\left(z^{k}\right)\right)^{N}\left(\phi\left(z^{N}\right)\right)^{k}=0$, so $\phi\left(z^{k}\right)=$ 0 hence $\phi$ is point evaluation at the point 0 . If $\phi\left(z^{N}\right) \neq 0$, let $a=$ $\phi\left(z^{N+1}\right) / \phi\left(z^{N}\right)$. For $k \in \mathbf{N}$ such that $z^{k} \in A$ we have $\phi\left(z^{k}\right) \cdot \phi\left(z^{N k}\right)=$ $\phi\left(z^{(N+1) k}\right)$ so $\phi\left(z^{k}\right)=a^{k}$, hence $\phi$ is point evaluation at $a \in D$.)

Since $a_{n}$ is an isolated point of the peak set $L$, by Rossi's local peak set theorem [2, p. 91] there exists $f_{n} \in[A, \bar{f} ; D]$ such that $f_{n}(x)=1$ for each $x \in L \backslash\left\{a_{n}\right\}$ and such that $\left|f_{n}(x)\right|<1$ for each $x \in\left\{a_{n}\right\} \cup D \backslash L$. Since $[A, \bar{f} ; D] \mid L$ is closed we may assume $f_{n}\left(a_{n}\right)=0[3$, p. 164]. So the maximal set $K$ of antisymmetry reduces to a single point $\left\{a_{n}\right\}$ or else $K \subset P$.

Now $P=L \cap \cap_{n=1}^{\infty}\left\{x \in D: f_{n}(x)=1\right\}$ is again a peak set for $[A, \bar{f} ; D]$ and is convex relative to the algebra $A$ (since $L$ is). So $\Delta[[A, \bar{f} ; D] \mid P]=$ $\Delta[A \mid P]=P$. Since $z^{n}$ and $z^{m}$ have no zeros on $P, z^{-n}, z^{-m} \in[[A, \bar{f} ; D] \mid P]$, so $[[A, \bar{f} ; D] \mid P]=C(P)$ and since $[A, \bar{f} ; D] \mid P$ is closed in $C(P)$ we have $[A, \bar{f} ; D] \mid P=C(P)$. So $[A, \bar{f} ; D] \mid K=C(K)$. By the Stone-Weierstrass theorem it follows that $[A, \bar{f} ; D]=C(D)$.

TheOREM 8 (MinSKer [5]). Let $n, m \in N$ such that $\operatorname{gcd}(n, m)=1$ and let $X$ be a compact subset of $\mathrm{C}$. Then $\left[z^{n}, \bar{z}^{m} ; X\right]=C(X)$.

Proof. $\operatorname{Re} z^{n m}$ and $\operatorname{Im} z^{n m}$ belong to $A=\left[z^{n}, \bar{z}^{m} ; X\right]$, so a maximal set of antisymmetry for $A$ consists of a finite number of points, so has to be a singleton. By the Stone-Weierstrass theorem $A=C(X)$.

We conclude with two questions. Let $D=\{|z| \leqslant 1\}$ and suppose that $f \in P(D)$ and $z^{2}$ separate the points of $D$. What can be said about the algebra $\left[z^{2}, \bar{f} ; D\right]$ ?

Let $X=\{1 \leqslant|z| \leqslant 2\}$. If $f \in R(X)$ and $z^{2}$ separate points of $X$, determine $\left[z^{2}, z^{-2}, \bar{f} ; X\right]$.

\section{REFERENCES}

1. J.-E. Björk and P. J. de Paepe, Maximal subalgebras, Papers from the Summer Gathering on Function Algebras at Aarhus, July 1969, Various Publications Series, No. 9, Matematisk Institut, Aarhus Universitet, Aarhus, 1969, pp. 36-39.

2. T. W. Gamelin, Uniform algebras, Prentice-Hall, Englewood Cliffs, N. J., 1969.

3. G. M. Leibowitz, Lectures on complex function algebras, Scott, Foresman and Co., Glenview, Ill., 1970.

4. S. N. Mergelyan, Uniform approximations to functions of a complex variable, Amer. Math. Soc. Transl. (1) 3 (1962), 281-391.

5. S. Minsker, Some applications of the Stone-Weierstrass Theorem to planar rational approximation, Proc. Amer. Math. Soc. 58 (1976), 94-96.

6. K. J. Preskenis, Approximation on disks, Trans. Amer. Math. Soc. 171 (1972), 445-467.

InSTITUUT voOR Propedeutische Wiskunde, UNIVERSITEIT VAN AMSTERDAM, AMSTERDAM, THE NETHERLANDS 